R. A. Blumenthal

Nagoya Math. J.

Vol. 90 (1983), 145-153

\title{
RIEMANNIAN FOLIATIONS WITH PARALLEL CURVATURE
}

\author{
ROBERT A. BLUMENTHAL
}

\section{§1. Introduction}

Let $M$ be a smooth compact manifold and let $\mathscr{F}$ be a smooth codimension $q$ Riemannian foliation of $M$. Let $T(M)$ be the tangent bundle of $M$ and let $E \subset T(M)$ be the subbundle tangent to $\mathscr{F}$. We may regard the normal bundle $Q=T(M) / E$ of $\mathscr{F}$ as a subbundle of $T(M)$ satisfying $T(M)=E \oplus Q$. Let $g$ be a smooth Riemannian metric on $Q$ invariant under the natural parallelism along the leaves of $\mathscr{F}$. This is equivalent to the existence of a bundle-like metric [16] and to the existence of a transverse $O(q)$-structure [5]. Recall that a connection $\nabla$ on $Q$ is basic if the induced parallel translation along a path lying in a leaf of $\mathscr{F}$ agrees with the natural parallelism along the leaves and that such a connection is characterized by the condition that $\nabla_{X} Y=[X, Y]_{Q}$ for all vector fields $X$ tangent to $E$ and $Y$ tangent to $Q$ where $[X, Y]_{Q}$ denotes the $Q$-component of the Lie bracket of $X$ and $Y$ [3]. The torsion of $V$ is the tensor field of type $(1,2)$ on $M$ defined by $T(X, Y)=\nabla_{X} Y_{Q}-\nabla_{Y} X_{Q}-[X, Y]_{Q}$ where $X$ and $Y$ are vector fields on $M$. There is a unique torsion-free metricpreserving basic connection $\nabla$ on $Q$ [9], [11] defined as follows. Let $x \in M$. Let $f: U \rightarrow V$ be a submersion whose level sets are the leaves of $\mathscr{F} \mid U$ where $U$ is a neighborhood of $x$ in $M$ and $V$ is an open set in $R^{q}$. There is a unique Riemannian metric $\bar{g}$ on $V$ such that $f^{*}(\bar{g})=g \mid U$. Let $\bar{\nabla}$ be the Riemannian connection on $V$. Then $\nabla \mid U=f^{-1}(\bar{\nabla})$. It is natural to study the relationship between the curvature of $\nabla$ and the structure of the foliated manifold $(M, \mathscr{F})$.

In the present work we study the case of parallel curvature, that is $\nabla R=0$ where $R(X, Y) Z$ denotes the curvature tensor of $\nabla$.

Let $\mathscr{F}$ be a Riemannian foliation with parallel curvature of a compact manifold $M$.

TheOREM 1. Let $\tilde{M}$ be the universal cover of $M$ and let $\tilde{\mathscr{F}}$ be the lift Received March 15, 1982. 
of $\mathscr{F}$ to $\tilde{M}$. Then $\tilde{M}$ fibers over a simply connected Riemannian symmetric space with the leaves of $\tilde{\mathscr{F}}$ as fibers.

Let $p \in M$. Let $\pi_{p}$ be a two-dimensional subspace of $Q_{p}$ and let $\{X, Y\}$ be an orthonormal basis of $\pi_{p}$. The (transverse) sectional curvature of $\pi_{p}$ is defined by $K\left(\pi_{p}\right)=-g_{p}(R(X, Y) X, Y)$ and depends only on $\pi_{p}$. If $K\left(\pi_{p}\right)>0$ (respectively, $\leq 0, \geq 0$ ) for all two-dimensional subspaces $\pi_{p} \subset$ $Q_{p}$ and all $p \in M$, we say that $(M, \mathscr{F})$ has positive (respectively, non-positive, non-negative) sectional curvature.

Corollary 1. If $(M, \mathscr{F})$ has non-positive sectional curvature, then $\tilde{M}$ is diffeomorphic to a product $\tilde{L} \times R^{q}$ where $\tilde{L}$ is the (common) universal cover of the leaves of $\mathscr{F}$ and the leaves of $\tilde{F}$ are identified with the sets $\tilde{L} \times\{x\}, x \in R^{q}$.

Corollary 2. If $\pi_{1}(M)$ is finite, then $(M, \mathscr{F})$ has non-negative sectional curvature and all the leaves of $\mathscr{F}$ are compact with finite holonomy.

Remark. If $\mathscr{F}$ is a flat Riemannian foliation of the compact manifold $M$, then $(M, \mathscr{F})$ has zero curvature and zero sectional curvature and so, by Corollary $1, \tilde{M} \cong \tilde{L} \times R^{q}$ and $\tilde{\mathscr{F}}$ is the product foliation. A theorem of G. Reeb [14] states that if $\mathscr{F}$ is a codimension one foliation of a compact manifold $M$ defined by a closed nonsingular one-form, then $\tilde{M} \cong \tilde{L} \times R$ and $\tilde{F}$ is the product foliation. It is easy to see that such a codimension one foliation admits a flat Riemannian structure and so we obtain Reeb's theorem from Corollary 1.

A differential $r$-form $\omega$ on $M$ is called base-like if on each coordinate neighborhood $U$ with coordinates $\left(x^{1}, \cdots, x^{k}, y^{1}, \cdots, y^{q}\right)$ respecting the foliation $\mathscr{F}$, the local expression of $\omega$ is of the form

$$
\sum_{1 \leq i_{1}<\cdots<i_{r} \leq q} a_{i_{1}, \cdots, i_{r}}\left(y^{1}, \cdots, y^{q}\right) d y^{i_{1}} \wedge \cdots \wedge d y^{i_{r}}
$$

[16], [17]. Since the exterior derivative of a base-like form is again baselike, one can construct the base-like cohomology algebra $H^{*}(M, \mathscr{F})=$ $\sum_{r \geq 0} H^{r}(M, \mathscr{F})$ of the foliated manifold $(M, \mathscr{F})$. For each $r \geq 0$, let $\beta_{r}(M, \mathscr{F})$ be the dimension of $H^{r}(M, \mathscr{F})$.

Theorem 2. If $(M, \mathscr{F})$ has positive sectional curvature, then $\beta_{1}(M, \mathscr{F})$ $=0$.

Recall from [13] the definition of the growth of a leaf $L$ of $\mathscr{F}$. Pick a Riemannian metric on $M$ (bundle-like or not) and restrict to obtain a 
Riemannian metric on $L$. Let $p \in L$ and define the growth function of $L$ at $p$ by $g_{p}(r)=\operatorname{vol}\left(B_{p}(r)\right)$ where $B_{p}(r)$ denotes the open ball in $L$ of radius $r$ centered at $p$. The growth type of $L$ is then defined to be the growth type of the function $g_{p}: R^{+} \rightarrow R^{+}$and is independent of the choice of metric on $M$ and of $p \in L$ [13].

TheOREm 3. The growth type of each leaf of $\mathscr{F}$ is dominated by the growth type of $\pi_{1}(M)$.

\section{§ 2. Proofs}

Let $\pi: F(Q) \rightarrow M$ be the frame bundle of $Q$, let $\omega$ be the connection form on $F(Q)$ associated to $\nabla$, and let $H \subset T(F(Q))$ be the corresponding horizontal distribution. Let $\left\{\left(U_{\alpha}, f_{\alpha}, g_{\alpha \beta}\right)\right\}_{\alpha, \beta \in A}$ be an $R^{q}$-cocycle defining $\mathscr{F}$. Let $F\left(R^{q}\right)$ be the frame bundle of $R^{q}$. Then $\left\{\left(\pi^{-1}\left(U_{\alpha}\right), f_{\alpha_{*}}, g_{\alpha \beta_{*}}\right)\right\}_{\alpha, \beta \in A}$ is an $F\left(R^{q}\right)$-cocycle on $F(Q)$ and defines a codimension $q(q+1)$ foliation $\mathscr{F}^{\prime}$ of $F(Q)$. Let $E^{\prime} \subset T(F(Q))$ be the integrable distribution whose integral manifolds are the leaves of $\mathscr{F}^{\prime}$. Since $\nabla$ is basic we have $E^{\prime} \subset H$ [12]. Let $\theta$ be the $R^{q}$-valued one-form on $F(Q)$ defined by $\theta_{u}(Y)=u^{-1}\left(\pi_{*_{u}}(Y)_{Q}\right)$ for $u \in F(Q), Y \in T_{u}(F(Q))$ where $u: R^{q} \rightarrow Q_{\pi(u)}$ denotes the vector space isomorphism which sends the standard basis of $R^{q}$ to the frame $u$ of $Q_{\pi(u)}$. The torsion form of $\nabla$ is the $R^{q}$-valued two-form $\Theta$ on $F(Q)$ defined by $\Theta_{u}(X, Y)=(d \theta)_{u}\left(X_{H}, Y_{H}\right)$ for $u \in F(Q)$ and $X, Y \in T_{u}(F(Q))$. Since $\nabla$ is the Riemannian basic connection, we have $\Theta=0$.

For each $h \in R^{q}$ let $B(h)$ be the unique horizontal vector field on $F(Q)$ satisfying $\pi_{*_{u}}\left(B(h)_{u}\right)=u(h)$ for all $u \in F(Q)$. Let $E^{i}=B\left(e_{i}\right)$ for $i=$ $1, \cdots, q$ where $\left\{e_{1}, \cdots, e_{q}\right\}$ is the standard basis of $R^{q}$ and let $Q^{\prime} \subset T(F(Q))$ be the $q$-plane bundle spanned by $E^{1}, \cdots, E^{q}$. Then $H=E^{\prime} \oplus Q^{\prime}$ and so $T(F(Q))=E^{\prime} \oplus Q^{\prime} \oplus V$ where $V$ is the bundle of vertical vectors. Hence we may regard $Q^{\prime} \oplus V$ as the normal bundle of $\mathscr{F}^{\prime}$. Let $E_{h}^{k}$ be the $q \times q$ matrix with a 1 in the $h^{\text {th }}$ column and $k^{\text {th }}$ row and 0 elsewhere and let $\sigma\left(E_{h}^{k}\right)$ be the corresponding fundamental vector field on $F(Q)$. Then $\left\{E^{i}\right.$, $\left.\sigma\left(E_{h}^{k}\right): i, h, k=1, \cdots, q\right\}$ is a trivialization of the normal bundle of $\mathscr{F}^{\prime}$. Recall that a vector field $Y$ on $F(Q)$ which is normal to $\mathscr{F}^{\prime}$ is said to be parallel along the leaves to $\mathscr{F}^{\prime}$ if $\left(f_{\alpha_{*}}\right)_{*}\left(Y \mid \pi^{-1}\left(U_{\alpha}\right)\right)$ is a well-defined vector field on $f_{\alpha_{*}}\left(\pi^{-1}\left(U_{\alpha}\right)\right) \subset F\left(R^{q}\right)$ for each $\alpha \in A$. This is equivalent to $Y$ being invariant under the natural parallelism along the leaves and is characterized by the condition that $[X, Y]$ is tangent to $\mathscr{F}^{\prime}$ whenever $X$ is a vector field tangent to $\mathscr{F}^{\prime}$ [5]. Since the fundamental vector fields 
on $F\left(R^{q}\right)$ are preserved by the maps $g_{\alpha \beta_{*}}$, it follows that the vector fields $\sigma\left(E_{h}^{k}\right)$ are parallel along the leaves to $\mathscr{F}^{\prime}$. Since $\nabla$ is the Riemannian basic connection, it is transversely projectable and hence $\left[X, E^{i}\right]$ is tangent to $\mathscr{F}^{\prime}$ for $i=1, \cdots, q$ whenever $X$ is a vector field tangent to $\mathscr{F}^{\prime}$ [10], and so $E^{1}, \cdots, E^{q}$ are parallel along the leaves to $\mathscr{F}^{\prime}$. Hence $\left\{E^{i}, \sigma\left(E_{h}^{k}\right)\right.$ : $i, h, k=1, \cdots, q\}$ is an $e$-structure for $\mathscr{F}^{\prime}$ [5].

Let $u \in F(Q)$ and let $X \in T_{u}(F(Q))$. Then there is a unique expression $X=X_{E^{\prime}}+X_{Q^{\prime}}+X_{V}$. Thus $\theta_{u}(X)=\theta_{u}\left(X_{Q^{\prime}}\right)=\theta_{u}\left(B(h)_{u}\right)=h$ for some $h \in R^{q}$ and so $X_{Q^{\prime}}=B\left(\theta_{u}(X)\right)_{u}$. Also $\omega_{u}(X)=\omega_{u}\left(X_{V}\right)=\omega_{u}\left(\sigma(A)_{u}\right)=A$ for some $A \in \operatorname{gl}(q, R)$ and so $X_{V}=\sigma\left(\omega_{u}(X)\right)_{u}$. Letting $X=\left[E^{i}, E^{j}\right]_{u}$, we obtain

$$
\left[E^{i}, E^{j}\right]_{Q_{u}}=B\left(\theta_{u}\left(\left[E^{i}, E^{j}\right]_{u}\right)\right)_{u} \text { and }\left[E^{i}, E^{j}\right]_{V u}=\sigma\left(\omega_{u}\left(\left[E^{i}, E^{j}\right]_{u}\right)\right)_{u} \text {. }
$$

Since

$$
\begin{aligned}
-\theta_{u}\left(\left[E^{i}, E^{j}\right]_{u}\right) & =E_{u}^{i} \theta\left(E^{j}\right)-E_{u}^{j} \theta\left(E^{i}\right)-\theta_{u}\left(\left[E^{i}, E^{j}\right]_{u}\right) \\
& =(d \theta)_{u}\left(E_{u}^{i}, E_{u}^{j}\right)=\Theta_{u}\left(E_{u}^{i}, E_{u}^{j}\right)=0,
\end{aligned}
$$

we have $\left[E^{i}, E^{j}\right]_{Q^{\prime}}=0$. Let $\Omega$ be the curvature form of $\nabla$ and write $\Omega=$ $\sum_{h, k=1}^{q} \Omega_{k}^{h} E_{h}^{k}$ where the $\Omega_{k}^{h}$ are two-forms on $F(Q)$. Since

$$
\begin{aligned}
-\omega_{u}\left(\left[E^{i}, E^{j}\right]_{u}\right) & =E_{u}^{i} \omega\left(E^{j}\right)-E_{u}^{j} \omega\left(E^{i}\right)-\omega_{u}\left(\left[E^{i}, E^{j}\right]_{u}\right) \\
& =(d \omega)_{u}\left(E_{u}^{i}, E_{u}^{j}\right)=\Omega_{u}\left(E_{u}^{i}, E_{u}^{j}\right),
\end{aligned}
$$

we have that

$$
\left[E^{i}, E^{j}\right]_{V}=-\sum_{h, k=1}^{q} \Omega_{k}^{h}\left(E^{i}, E^{j}\right) \sigma\left(E_{h}^{k}\right) .
$$

Let $u_{0} \in F(Q)$ and let $P\left(u_{0}\right)=\left\{u \in F(Q): u\right.$ can be joined to $u_{0}$ by a horizontal curve\}, the holonomy bundle through $u_{0}$. Let $u \in P\left(u_{0}\right)$ and let $p=\pi(u) \in M$. Let $c:(-\varepsilon, \varepsilon) \rightarrow M$ be a smooth curve with $c(0)=p$, and let $c^{*}:(-\varepsilon, \varepsilon) \rightarrow F(Q)$ be the unique horizontal lift of $c$ satisfying $c^{*}(0)$ $=u$. Fix $1 \leq i, j, l \leq q$ and let $X(t)=c^{*}(t)_{i}, \quad Y(t)=c^{*}(t)_{j}$, and $Z(t)=$ $c^{*}(t)_{l}$. Then $X, Y$ and $Z$ are normal vector fields along $c$ which are parallel along $c$ and hence, since $R$ is parallel, $\nabla_{\dot{c}(0)} R(X, Y) Z=0$. Defining $f:(-\varepsilon, \varepsilon) \rightarrow R^{q}$ by $f(t)=c^{*}(t)^{-1}(R(X, Y) Z)_{c(t)}$, we have that $\nabla_{c(0)} R(X, Y) Z$ $=u\left(f^{\prime}(0)\right)$ and so $f^{\prime}(0)=0$. But

$$
(R(X, Y) Z)_{c(t)}=c^{*}(t)\left(\Omega_{c^{*}(t)}\left(E_{c^{*}(t)}^{i}, E_{c^{*}(t)}^{j}\right) c^{*}(t)^{-1}\left(c^{*}(t)_{l}\right)\right)
$$

and hence $f(t)=\Omega_{c^{*}(t)}\left(E_{c^{*}(t)}^{i}, E_{c^{*}(t)}^{j}\right) \cdot e_{l}=l^{\text {th }}$ column of $\Omega_{c^{*}(t)}\left(E_{c^{*}(t)}^{i}, E_{c^{*}(t)}^{j}\right)$. Thus $\dot{c}^{*}(0)\left(l^{\text {th }}\right.$ column of $\left.\Omega\left(E^{i}, E^{j}\right)\right)=f^{\prime}(0)=0$ and hence 


$$
W\left(l^{\text {th }} \text { column of } \Omega\left(E^{i}, E^{j}\right)\right)=0
$$

for all $W \in H_{u}$. Thus $\Omega_{k}^{h}\left(E^{i}, E^{j}\right)$ is constant on $P\left(u_{0}\right)$ for each $1 \leq h, k \leq q$. Let $\Phi\left(u_{0}\right)$ be the holonomy group with reference point $u_{0}$; that is, $\Phi\left(u_{0}\right)=\left\{A \in \operatorname{gl}(q, R): u_{0}\right.$ and $u_{0} A$ can be joined by a horizontal curve $\}$. Then $P\left(u_{0}\right)$ is a reduced bundle with structure group $\Phi\left(u_{0}\right)$ such that the natural parallelism along the leaves of $\mathscr{F}$ carries elements of $P\left(u_{0}\right)$ to elements of $P\left(u_{0}\right)$ and so $P\left(u_{0}\right)$ is a transverse $\Phi\left(u_{0}\right)$-structure for $\mathscr{F}$ [5] and $\omega$ is reducible to a basic connection in $P\left(u_{0}\right)$. Let $V^{\prime} \subset T\left(P\left(u_{0}\right)\right)$ be the subbundle consisting of vectors tangent to the fibers of $P\left(u_{0}\right)$. Then $T\left(P\left(u_{0}\right)\right)=E^{\prime} \oplus Q^{\prime} \oplus V^{\prime}$ and $\mathscr{F}^{\prime}$ is a foliation of $P\left(u_{0}\right)$ whose tangent bundle is $E^{\prime}$. Let $A_{1}, \cdots, A_{r}$ be a basis of the Lie algebra of $\Phi\left(u_{0}\right)$. Then $\left\{E^{1}, \cdots, E^{q}, \sigma\left(A_{1}\right), \cdots, \sigma\left(A_{r}\right)\right\}$ is an $e$-structure for $\mathscr{F}^{\prime}$ on $P\left(u_{0}\right)$. On $P\left(u_{0}\right)$ we have

$$
\begin{aligned}
& {\left[E^{i}, E^{j}\right]_{Q^{\prime}}=0} \\
& {\left[E^{i}, E^{j}\right]_{V^{\prime}}=\sum_{k=1}^{r} f_{i j}^{k} \sigma\left(A_{k}\right)} \\
& {\left[\sigma\left(A_{i}\right), \sigma\left(A_{j}\right)\right]=\sum_{k=1}^{r} c_{i j}^{k} \sigma\left(A_{k}\right)} \\
& {\left[\sigma\left(A_{i}\right), E^{j}\right]=B\left(A_{i} \cdot e_{j}\right)=\sum_{k=1}^{q} b_{i j}^{k} E^{k}}
\end{aligned}
$$

where $f_{i j}^{k}, c_{i j}^{k}$, and $b_{i j}^{k}$ are constants.

Let $G$ be the unique simply connected Lie group with Lie algebra $g$ spanned by elements $Z_{1}, \cdots, Z_{q}, B_{1}, \cdots, B_{r}$ satisfying

$$
\begin{aligned}
& {\left[Z_{i}, Z_{j}\right]=\sum_{k=1}^{r} f_{i j}^{k} B_{k}} \\
& {\left[B_{i}, B_{j}\right]=\sum_{k=1}^{r} c_{i j}^{k} B_{k}} \\
& {\left[B_{i}, Z_{j}\right]=\sum_{k=1}^{q} b_{i j}^{k} Z_{k} .}
\end{aligned}
$$

Let $h$ be the subalgebra of $g$ spanned by $B_{1}, \cdots, B_{r}$ and let $m$ be the subspace of $g$ spanned by $Z_{1}, \cdots, Z_{q}$. Then $g=h \oplus m,[h, h] \subset h,[h, m] \subset m$, and $[m, m] \subset h$. Let $X \in g$ and write $X$ uniquely as $X=Y+Z$ where $Y \in h$, $Z \in m$. Let $\tau(X)=Y-Z$. Then $\tau$ is an automorphism of $g$ and $\tau^{2}$ is the identity. Since $G$ is simply connected there is an automorphism $F: G \rightarrow G$ such that $F_{*}=\tau$. Let $H$ be the identity component of the subgroup of $G$ fixed by $F$. Then $H$ is a closed Lie subgroup of $G$ and the triple $(G, H, F)$ is a symmetric space. 
Let $u \in P\left(u_{0}\right)$. Since $E^{1}, \cdots, E^{q}, \sigma\left(A_{1}\right), \cdots, \sigma\left(A_{r}\right)$ are parallel along the leaves to $\mathscr{F}^{\prime}$, there is a neighborhood $W$ of $u$ in $P\left(u_{0}\right)$ and a smooth submersion $\bar{f}: W \rightarrow G$ such that

$$
\begin{aligned}
& \operatorname{kernel}\left(\bar{f}_{*_{y}}\right)=E_{y}^{\prime} \\
& \bar{f}_{*_{y}}\left(E_{y}^{i}\right)=Z_{i \bar{f}(y)}, i=1, \cdots, q \\
& \bar{f}_{*_{y}}\left(\sigma\left(A_{j}\right)_{y}\right)=B_{\bar{j}^{\prime}(y)}, j=1, \cdots, r
\end{aligned}
$$

for all $y \in W$. Let $U=\pi(W)$, a neighborhood of $\pi(u)$ in $M$. Then $\bar{f}$ induces a smooth submersion $f: U \rightarrow G / H$ such that kernel $\left(f_{*_{p}}\right)=E_{p}$ for all $p \in U$ and the diagram

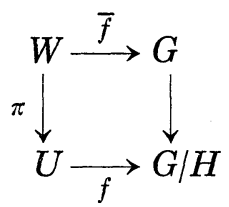

commutes. Let $\bar{\theta}$ be the canonical left-invariant $g$-valued one-form on $G$ and let $\bar{\theta}_{h}$ be the $h$-component of $\bar{\theta}$. Then $\bar{\theta}_{h}$ defines a $G$-invariant connection in the principal $H$-bundle $G \rightarrow G / H$ which induces the canonical linear connection on the symmetric space $G / H[8]$, and $\bar{f}^{*} \bar{\theta}_{h}=\omega$. Thus if $F(G / H)$ is the frame bundle of $G / H$ and $\bar{\omega}$ is the connection form on $F(G / H)$ corresponding to the canonical linear connection on $G / H$, we have that $\left(f_{*}\right)^{*} \bar{\omega}=\omega$ on $\left.F(Q)\right|_{U}$. Thus we can find a $G / H$-cocycle $\left\{\left(U_{\alpha}, f_{\alpha}, g_{\alpha \beta}\right)\right\}_{\alpha, \beta \in A}$ defining $\mathscr{F}$ such that $\left(f_{\alpha_{*}}\right) * \bar{\omega}=\omega$ on $\left.F(Q)\right|_{U_{\alpha}}$. If $U_{\alpha} \cap U_{\beta} \neq \phi$ then, since $\left(f_{\alpha_{*}}\right) * \bar{\omega}=\omega=\left(f_{\beta_{*}}\right) * \bar{\omega}$ on $\left.F(Q)\right|_{U_{\alpha} \cap U_{\beta}}$, it follows that $\left(g_{\alpha \beta_{*}}\right) * \bar{\omega}=\bar{\omega}$ on $\left.F(G / H)\right|_{f_{\beta}\left(U_{\alpha} \cap U_{\beta}\right)}$. Without loss of generality we may assume that $U_{\alpha} \cap U_{\beta}$ is connected whenever it is non-empty. Hence, since $\bar{\omega}$ is a complete analytic linear connection on the simply connected analytic manifold $G / H, g_{\alpha \beta}$ extends to an affine isomorphism of $G / H$ [8].

Hence $\mathscr{F}$ is transversely homogeneous. The foliated manifold $(\tilde{M}, \tilde{F})$ admits a complete bundle-like metric, and so we have that $\tilde{\mathscr{F}}$ is regular [1]. Hence the space of leaves $\tilde{M} / \tilde{F}$ is a complete, Riemannian, Hausdorff manifold and the natural projection $f: \tilde{M} \rightarrow \tilde{M} / \tilde{F}$ is a fibration [16]. Let $N$ denote the Riemannian manifold $\tilde{M} / \tilde{\mathscr{F}}$. Since the metric on $N$ is induced by the bundle-like metric on $\tilde{M}$, it follows that the curvature tensor field of $N$ is parallel. Thus $N$ is a complete, simply connected, Riemannian locally symmetric space and hence is Riemannian symmetric [8] and so Theorem 1 is proved. 
If $(M, \mathscr{F})$ has non-positive sectional curvature, then $N$ has non-positive sectional curvature. Since $N$ is complete and simply connected we have that $N$ is diffeomorphic to $R^{q}[8]$ and hence the fibration $f: \tilde{M} \rightarrow N$ is a product, proving Corollary 1. See [2] for similar results in the flat nonRiemannian case.

If $\pi_{1}(M)$ is finite, then $\tilde{M}$ is compact and so the leaves of $\tilde{F}$ are compact. Thus all the leaves of $\mathscr{F}$ are compact. Hence $\mathscr{F}$ is a closed metric foliation and so the holonomy group of each leaf is finite [15]. Since $N$ is compact it has non-negative sectional curvature [18] and so $(M, \mathscr{F})$ has non-negative sectional curvature, proving Corollary 2.

We now prove Theorem 2. Each covering transformation $\sigma$ of $\tilde{M}$ induces an isometry $\Psi(\sigma)$ of $N$. We thus obtain a homomorphism $\Psi: \pi_{1}(M) \rightarrow I(N)$ where $I(N)$ denotes the isometry group of $N$ such that $\Psi(\sigma) \circ f=f \circ \sigma$ for all $\sigma \in \pi_{1}(M)$. Let $\Sigma$ be the image of $\Psi$ in $I(N)$ and let $K$ be the closure of $\Sigma$. For each $r \geq 0$ let $A_{K}^{r}(N)$ be the space of $K$-invariant $r$-forms on $N$ and let $A^{r}(M, \mathscr{F})$ be the space of base-like $r$-forms on $M$. Let $\eta \in A_{K}^{r}(N)$. Then $f^{*} \eta$ is an $r$-form on $\tilde{M}$ which is base-like with respect to $\tilde{\mathscr{F}}$. Since $\eta$ is $\Sigma$-invariant it follows that $f^{*} \eta$ is $\pi_{1}(M)$-invariant and hence there exists a unique $\omega \in A^{r}(M, \mathscr{F})$ such that $f^{*} \eta=p^{*} \omega$ where $p: \tilde{M} \rightarrow M$ is the covering projection. Conversely, suppose $\omega \in A^{r}(M, \mathscr{F})$. Then $p^{*} \omega$ is base-like and hence there exists a unique $r$-form $\eta$ on $N$ such that $p^{*} \omega=f^{*} \eta$. Since $p^{*} \omega$ is $\pi_{1}(M)$-invariant it follows that $\eta$ is $\Sigma$-invariant and hence $K$-invariant. Thus $\eta \in A_{K}^{r}(N)$. We have constructed an isomorphism of cochain complexes

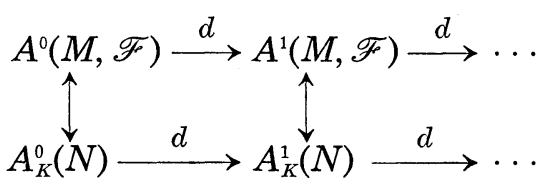

and hence we obtain an isomorphism in cohomology $H^{*}(M, \mathscr{F}) \rightarrow H_{K}^{*}(N)$. Since $(M, \mathscr{F})$ has positive sectional curvature it follows that $N$ has positive sectional curvature. Thus $N$ is compact [18] and so $K$ is compact. Hence the inclusion of the algebra of $K$-invariant forms on $N$ into the algebra of differential forms on $N$ induces an injection $H_{K}^{*}(N) \rightarrow H^{*}(N)$ [4], [6]. Thus $H^{*}(M, \mathscr{F})$ is isomorphic to a subalgebra of $H^{*}(N)$. Since $N$ is simply connected we have that $H^{1}(N)=0$ and hence $H^{1}(M, \mathscr{F})=0$.

Let $\Sigma \backslash N$ be the orbit space of $\Sigma$. To prove Theorem 3 we construct a map $h: M / \mathscr{F} \rightarrow \Sigma \backslash N$ and apply a result in [1]. Let $L$ be a leaf of $\mathscr{F}$. 
Choose a leaf $\tilde{L}$ of $\tilde{\mathscr{F}}$ such that $p(\tilde{L})=L$. Let $x=f(\tilde{L}) \in N$. Then the orbit of $x$ under $\Sigma$ depends only on $L$ and we denote it by $h(L)$. The growth type of a leaf $L$ of $\mathscr{F}$ is dominated by the growth type of $h(L)$ [1] which in turn is dominated by the growth type of $\Sigma$. Hence, since $\Sigma$ is a homomorphic image of $\pi_{1}(M)$, we have that the growth type of $L$ is dominated by the growth type of $\pi_{1}(M)$.

\section{§3. Examples}

EXAMPLE 1. Let $G$ be a compact connected Lie group of dimension $q$ and let $g$ be the Lie algebra of $G$. Let $M$ be a compact manifold and suppose $\omega$ is a smooth $g$-valued one-form of rank $q$ on $M$ satisfying $d \omega+\frac{1}{2}[\omega, \omega]=0$. Then $\omega$ defines a smooth codimension $q$ foliation $\mathscr{F}$ on $M$ which is a Lie foliation modeled on $G$ [7]. Let $\langle$,$\rangle be a bi-invariant$ Riemannian metric on $G$. Then $\langle$,$\rangle induces on (M, \mathscr{F})$ a Riemannian structure with parallel curvature and non-negative sectional curvature.

Example 2. Let $H$ be a Lie subgroup of the $n$-dimensional torus $T^{n}$. Then the foliation $\mathscr{F}$ of $T^{n}$ by the cosets of $H$ admits a Riemannian structure with vanishing curvature and $\beta_{1}\left(T^{n}, \mathscr{F}\right) \neq 0$.

ExAMPLE 3. Let $M$ be the unit tangent bundle of the two-holed torus $T_{2}$. Let $\mathscr{F}$ be the foliation of $M$ by the circle fibers. Then $\mathscr{F}$ admits a Riemannian structure with parallel curvature and negative sectional curvature and $\beta_{1}(M, \mathscr{F}) \neq 0$.

EXAMPLE 4. Let $\Phi: \pi_{1}\left(T_{2}\right) \rightarrow S O(3)$ be a homomorphism whose image is dense in $S O(3)$. This defines a left action of $\pi_{1}\left(T_{2}\right)$ on $S^{2}$. Let $H$ be the universal cover of $T_{2}$. Then $H$ is a principal $\pi_{1}\left(T_{2}\right)$-bundle over $T_{2}$. Let $M=H \times_{\pi_{1}\left(T_{2}\right)} S^{2}$ be the associated bundle with fiber $S^{2}$. The foliation of $H \times S^{2}$ whose leaves are the sets $H \times\{x\}, x \in S^{2}$ passes to a foliation $\mathscr{F}$ of $M$ all of whose leaves are dense. Since $\pi_{1}\left(T_{2}\right)$ acts on $S^{2}$ by isometries, $(M, \mathscr{F})$ admits a Riemannian structure with parallel curvature and positive sectional curvature. Also $\beta_{1}(M, \mathscr{F})=0$.

Example 5. Define a left action of the integers $Z$ on $S^{2}$ by letting the generator act as

$$
\left(\begin{array}{ccc}
\cos 2 \pi \alpha & \sin 2 \pi \alpha & 0 \\
-\sin 2 \pi \alpha & \cos 2 \pi \alpha & 0 \\
0 & 0 & 1
\end{array}\right)
$$


where $0<\alpha<1$ is irrational. Let $M=R \times{ }_{Z} S^{2}$. Then $M$ is an $S^{2}$-bundle over $S^{1}$ with a codimension 2 foliation $\mathscr{F}$. The foliated manifold $(M, \mathscr{F})$ admits a Riemannian structure with parallel curvature and positive sectional curvature and $\beta_{1}(M, \mathscr{F})=0$. There are exactly two compact leaves. If $L$ is a non-compact leaf then $\bar{L}$ is diffeomorphic to $T^{2}$. The foliation of $\bar{L}$ by the leaves of $\mathscr{F}$ is an irrational slope foliation and hence is a flat Riemannian foliation and $\beta_{1}(\bar{L}, \mathscr{F}) \neq 0$.

\section{REFERENCES}

[1] R. A. Blumenthal, Transversely homogeneous foliations, Ann. Inst. Fourier, 29 (1979), 143-158.

[2] — Foliated manifolds with flat basic connection, J. Differential Geom., 16 (1981), 401-406.

[2] R. Bott, Lectures on characteristic classes and foliations (notes by L. Conlon), Lecture Notes in Math., no. 279, Springer-Verlag, New York, 1972, 1-80.

[4.] C. Chevalley and S. Eilenberg, Cohomology theory of Lie groups and Lie algebras, Trans. Amer. Math. Soc., 63 (1948), 85-124.

[5] L. Conlon, Transversally parallelizable foliations of codimension two, Trans. Amer. Math. Soc., 194 (1974), 79-102.

[6] W. Greub, S. Halperin and R. Vanstone, Connections, curvature, and cohomology, vol. II, Academic Press, New York, 1973.

[ 7 ] R. Hermann, On the differential geometry of foliations, Ann. of Math., 72 (1960), $445-457$.

[8] S. Kobayashi and K. Nomizu, Foundations of differential geometry, vol. I, II. Interscience Tracts in Pure and Appl. Math., 15, Interscience, New York, 1963.

[ 9 ] C. Lazarov and J. Pasternack, Secondary characteristic classes for Riemannian foliations, J. Differential Geom., 11 (1976), 365-385.

[10] F. Molino, Connexions et G-structures sur les variétés feuilletées, Bull. Sci. Math., 92 (1968), 59-63.

[11] - Etude des feuilletages transversalement complets et applications, Ann. Sci. École Norm. Sup., 10 (1977), 289-307.

[12] — Feuilletages et classes caractéristiques, Symposia Mathematica, X (1972), 199-209.

[13] J. F. Plante, Foliations with measure preserving holonomy, Ann. of Math., 102 (1975), 327-361.

[14] G. Reeb, Sur certaines propriétés topologiques des variétés feuilletées, Actualités Sci. Indust., no. 1183, Hermann, Paris, 1952.

[15] B. Reinhart, Closed metric foliations, Michigan Math. J., 8 (1961), 7-9.

[16] — Foliated manifolds with bundle-like metrics, Ann. of Math., 69 (1959), 119 132.

[17] —-, Harmonic integrals on foliated manifolds, Amer. J. Math., 81 (1959), 529536.

[18] J. Wolf, Spaces of constant curvature, McGraw-Hill, New York, 1967.

Department of Mathematics

Saint Louis University

St. Louis, Missouri 63103 